\title{
Surgeon attitudes toward point of care ultrasound for biliary disease: a nationwide Canadian survey
}

\author{
Jennifer Koichopolos, MD \\ Richard Hilsden, MD, MBA \\ Frank Myslik, MD \\ Drew Thompson, MD \\ Jeremy Vandelinde, BSc \\ Rob Leeper, MD, MEHP
}

Accepted Apr. 24, 2019

\section{Correspondence to:}

W.R. Leeper

Department of Surgery

Western University

Rm E2-215

London Health Sciences Centre

Victoria Hospital

800 Commissioners Rd East

London ON N6A 5W9

rob.leeper@Ihsc.on.ca

DOI: $10.1503 . c j s / 010218$

\begin{abstract}
SUMMARY
Multiple studies confirm that point of care ultrasound (PoCUS) has a high sensitivity and specificity for cholelithiasis and cholecystitis. However, there is poor perceived reliability of biliary PoCUS by surgeons. This survey was performed to assess surgeons' opinions on using PoCUS in gallstone disease and barriers that exist for its institution. The majority $(60.3 \%)$ of respondents reported a total lack of confidence in PoCUS for the diagnosis of biliary disease. Most felt the sensitivity of PoCUS was poor and had concerns about the user-dependent nature of the test and the lack of imaging details provided. If offered ideal clinical/laboratory findings with PoCUS results, only $4.7 \%$ of surgeons would definitely operate for unremitting biliary colic and 5.4\% for cholecystitis. The ability to replicate findings independently increased confidence in clinical decision-making. Our findings suggest there is substantial distrust in biliary PoCUS but that specific ultrasound training for the surgical workforce may prove tremendously beneficial for its utilization.
\end{abstract}

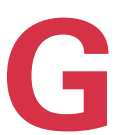
allstone disease affects more than 20 million Americans yearly. ${ }^{1}$ In Ontario, 260 elective cholecystectomies per 100000 patients are performed each year. ${ }^{2}$ Point of care ultrasound (PoCUS) has a growing role in the diagnosis of gallstone disease. ${ }^{3}$ Results of PoCUS are similarly accurate compared with formal radiology-performed ultrasounds. ${ }^{4}$ Emergency physicians who use biliary PoCUS improve emergency department (ED) throughput and reduce length of stay in the ED. ${ }^{3}$ However, surgeons still depend on formal radiology-performed imaging for the management of biliary disease. ${ }^{5}$

We surveyed surgeons about their opinions of biliary PoCUS. Our objectives were to quantify surgeon confidence in biliary PoCUS and elucidate possible barriers to use in order to improve surgeon utilization.

\section{OUR APPROACH}

A cross-sectional survey was emailed to general surgery program directors from the 14 primarily English-speaking academic programs in Canada. They were asked to distribute the survey to all general surgery residents, fellows and staff associated with their centre and to provide us with the number of survey recipients so we could determine the overall response rate.

To explore the factors associated with confidence in biliary PoCUS, we dichotomized confidence by considering everyone who answered "slightly confident" to "extremely confident" as having some confidence with PoCUS and those who answered "unconfident" as being unconfident with PoCUS. All other variables were dichotomized as seen in Box 1.

We also distilled 5 potential barriers to applying the results of biliary PoCUS in clinical practice: perceived poor test characteristics, user-dependent nature of the technology, poor documentation of results, lack of personal ability to replicate the test, and lack of detailed imaging findings. We analyzed the response data to model the relationships between the survey respondents' 
Box 1. Dichotomization of factors used in data analysis

\begin{tabular}{|c|c|c|c|c|c|c|}
\hline \multirow{2}{*}{$\begin{array}{l}\text { Factor } \\
\text { Level of training }\end{array}$} & \multicolumn{3}{|c|}{$\begin{array}{l}\text { Responses considered staff level, frequent, high } \\
\text { sensitivity or management altering }\end{array}$} & \multicolumn{3}{|c|}{$\begin{array}{l}\text { Responses considered trainee level, infrequent, } \\
\text { low sensitivity or not altering management }\end{array}$} \\
\hline & - & $\begin{array}{c}\text { Staff surgeon } \\
\text { (>10 years } \\
\text { practice) }\end{array}$ & $\begin{array}{c}\text { Staff surgeon } \\
\text { (<10 years } \\
\text { practice) }\end{array}$ & Fellow & $\begin{array}{l}\text { Senior resident } \\
\text { (years 3-5) }\end{array}$ & $\begin{array}{l}\text { Junior resident } \\
\text { (years 1-2) }\end{array}$ \\
\hline $\begin{array}{l}\text { Frequency that PoCUS results are } \\
\text { used in practice }\end{array}$ & Very often & Somewhat often & Occasionally & Rarely & Never & - \\
\hline $\begin{array}{l}\text { Frequency of performing PoCUS } \\
\text { personally in practice }\end{array}$ & Very often & Somewhat often & Occasionally & Rarely & Never & - \\
\hline $\begin{array}{l}\text { Frequency of referrals based on } \\
\text { PoCUS-diagnosed biliary disease }\end{array}$ & $>20$ times & 10-20 times & 5-10 times & $3-5$ times & $1-2$ times & Never \\
\hline $\begin{array}{l}\text { Perceived sensitivity of PoCUS for } \\
\text { cholelithiasis }\end{array}$ & $90 \%-100 \%$ & $80 \%-90 \%$ & $70 \%-80 \%$ & $60 \%-70 \%$ & $50 \%-60 \%$ & $<50 \%$ \\
\hline $\begin{array}{l}\text { Perceived sensitivity of PoCUS for } \\
\text { cholecystitis }\end{array}$ & $90 \%-100 \%$ & $80 \%-90 \%$ & $70 \%-80 \%$ & $60 \%-70 \%$ & $50 \%-60 \%$ & $<50 \%$ \\
\hline $\begin{array}{l}\text { Ability to admit, diagnose and/or } \\
\text { operate based on PoCUS findings }\end{array}$ & $\begin{array}{l}\text { Yes, } \\
\text { definitely }\end{array}$ & Likely yes & Maybe & Likely no & $\begin{array}{l}\text { No, definitely } \\
\text { not }\end{array}$ & - \\
\hline
\end{tabular}

attitudes and experience of PoCUS in diagnosis and management of biliary disease.

Eleven of 14 schools responded to our request to participate, totalling 132 general surgery staff and residents. The overall national response rate was $25.2 \%$. Responses indicated most surgeons had previous experience with PoCUS. Almost half had some form of PoCUS training (43.5\%). Most occasionally used the results of PoCUS (25.9\%), though they rarely/never personally performed the test (58\%). Surgeons were familiar with PoCUS done by emergency physicians (88.5\%), and most received 5-10 consults per year based on biliary PoCUS (23.7\%) (Fig. 1).

The majority $(60.3 \%)$ of respondents had a total lack of confidence in biliary PoCUS, and 26.7\% were slightly confident (Fig. 2).

Surgeons felt the test characteristics of PoCUS for cholelithiasis was modest (median $70 \%-80 \%$ ). Almost half (48.9\%) felt the sensitivity for cholecystitis was less than $50 \%$. Other barriers to using PoCUS included how the test is documented and the user-dependent nature of the test. Most felt that if they were able to perform biliary PoCUS themselves, their confidence would increase $(79.8 \%)$.

Hypothetical clinical decision-making using PoCUS in patients with classic biliary colic and cholecystitis also showed low confidence in the technology (Table 1).

On univariate analysis, multiple factors were found to significantly affect the confidence of surgeons using biliary PoCUS (Table 2). A regression analysis was additionally performed to assess which modifiable factors independently affected clinical decision-making. Ability to personally replicate the test independently affected a surgeon's willingness to diagnose unremitting biliary colic (odds ratio $[\mathrm{OR}] 3.57, p=0.02$ ), operate for unremitting biliary colic (OR 8.53, $p=0.05)$ and diagnose cholecystitis (OR 2.87, $p=0.05)$.

Differences between trainees and staff were evaluated. Residents more frequently used the results of

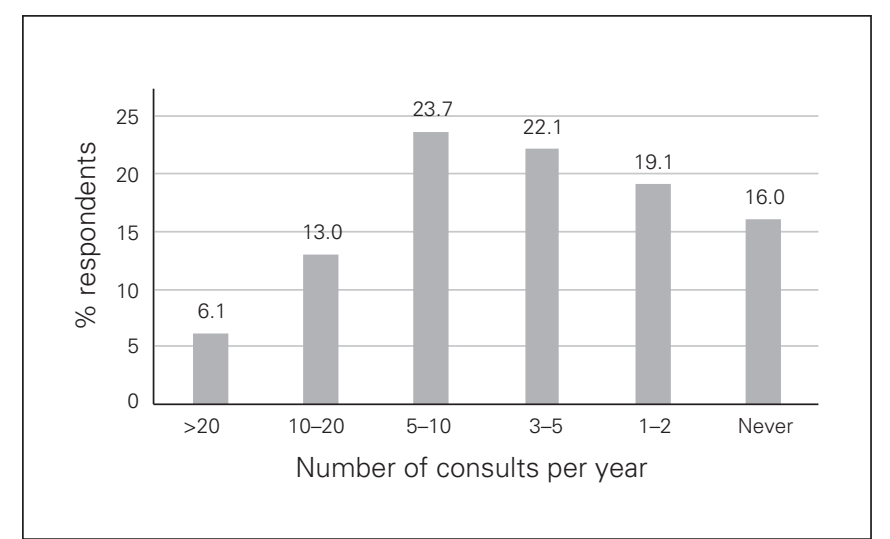

Fig. 1. Number of consults per year from emergency department on the basis of point of care ultrasound for suspected biliary disease.

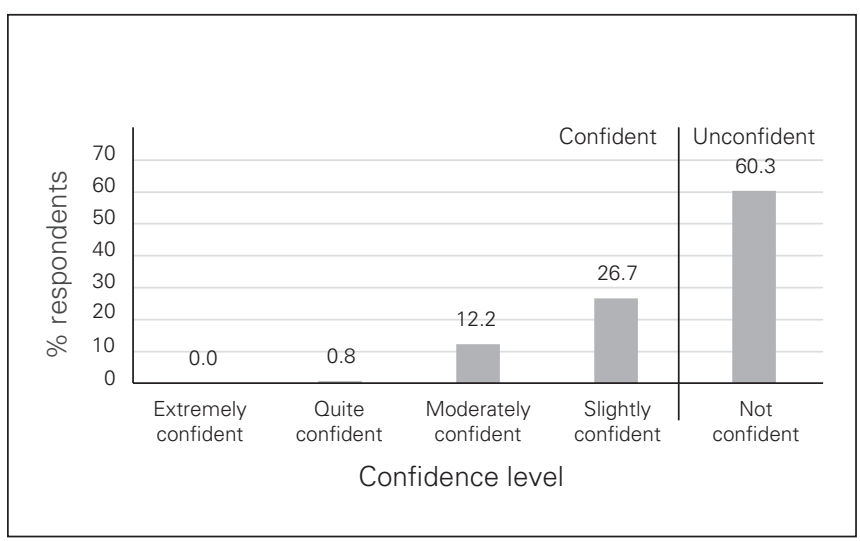

Fig. 2. Confidence in diagnostic information on the basis of point of care ultrasound in patients with biliary disease.

PoCUS $(74.0 \%$ v. $51.9 \%, p=0.01)$ and performed their own ultrasounds $(50.6 \%$ v. $29.6 \%, p=0.02)$. They were more aware of the ED use of PoCUS $(96.1 \%$ v. $77.8 \%$, $p=0.002)$ and received more referrals $(53.2 \%$ v. $27.8 \%$, 
Table 1. Clinical decision-making using biliary PoCUS

\begin{tabular}{|c|c|c|c|c|c|}
\hline \multirow[b]{2}{*}{ Scenario } & \multicolumn{5}{|c|}{ Decision; no. (\%) } \\
\hline & Yes, definitely & Likely yes & Maybe & Likely no & No, definitely not \\
\hline \multicolumn{6}{|c|}{$\begin{array}{l}\text { A patient presents with a history consistent with unremitting biliary colic and normal blood work. Is the finding of gallstones on } \\
\text { PoCUS enough for you to }\end{array}$} \\
\hline $\begin{array}{l}\text { Admit overnight for unremitting } \\
\text { biliary colic }\end{array}$ & $14(10.9)$ & $43(33.3)$ & $37(28.7)$ & $23(17.8)$ & $12(9.3)$ \\
\hline Diagnose biliary colic & $14(10.9)$ & $32(24.8)$ & $47(36.4)$ & $26(20.2)$ & $10(7.8)$ \\
\hline Operate for biliary colic & $6(4.7)$ & $9(7.0)$ & $15(11.7)$ & $31(24.2)$ & $67(52.3)$ \\
\hline \multicolumn{6}{|c|}{$\begin{array}{l}\text { A patient presents with elevated white count, RUQ pain and normal bilirubin. Are the findings of sonographic Murphy sign and } \\
\text { gallstones on PoCUS enough for you to }\end{array}$} \\
\hline Admit overnight for cholecystitis & $31(24.0)$ & $44(34.1)$ & $32(24.8)$ & $14(10.9)$ & $8(6.2)$ \\
\hline Diagnose cholecystitis & 15 (11.6) & $32(24.8)$ & $40(31.0)$ & $28(21.7)$ & $15(11.6)$ \\
\hline Operate for cholecystitis & $7(5.4)$ & $9(7.0)$ & 17 (13.3) & 37 (28.9) & $58(45.3)$ \\
\hline
\end{tabular}

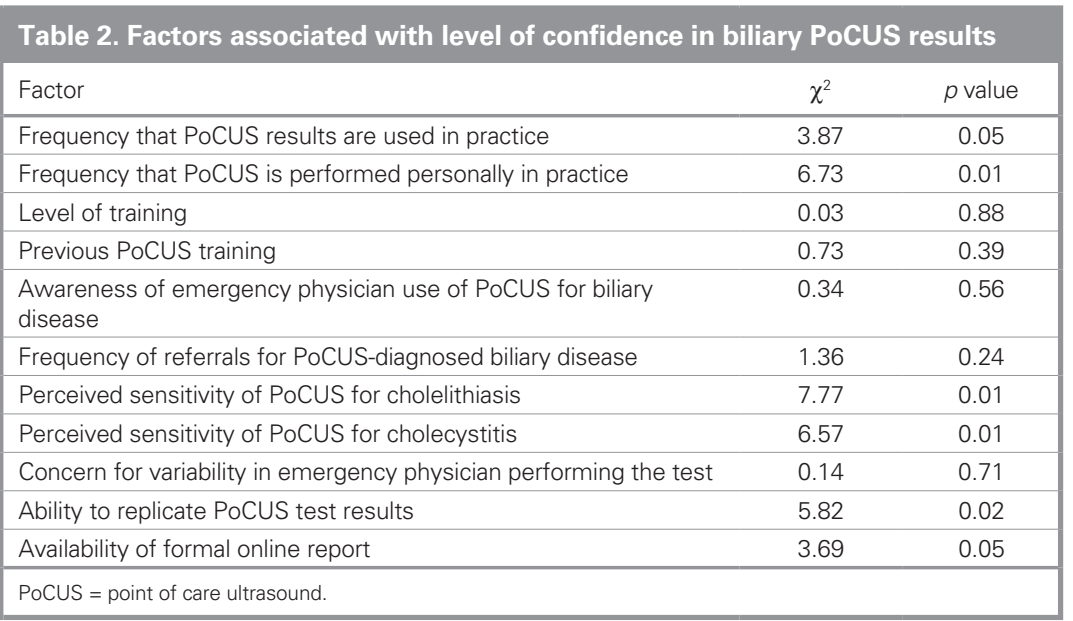

$p=0.004)$. Their confidence in the test was, however, the same $(40.2 \%$ v. $39.9 \%, p>0.99)$. Additionally, trainees were less willing to treat patients based on the results of biliary PoCUS.

\section{Discussion}

To our knowledge, we were the first to investigate how Canadian general surgeons are using biliary PoCUS results in their practice and the barriers to its adoption.

Currently, there is a lack of confidence in the results of biliary PoCUS. A potential source of hesitation among surgeons may be incomplete knowledge translation from the literature. Recent reviews have shown that PoCUS sensitivity for cholelithiasis and cholecystitis is $89.8 \%{ }^{5}$ and $87 \%$, respectively. ${ }^{4}$ In our survey, most surgeons believed the sensitivity for cholelithasis and cholecystitis was $70 \%-$ $80 \%$ and $<50 \%$, respectively.

This study identifies modifiable factors that may affect surgeons' confidence in biliary PoCUS. Education around the documented sensitivity of biliary PoCUS, training to replicate the test, and the availability of an online report would all be beneficial. The time commit- ment required to achieve this training is likely small, as evidence shows surgeons developed PoCUS skills quickly after a short didactic and hands-on education session. ${ }^{6}$

There were multiple differences between the responses of residents and staff. Trainees appeared to have greater exposure to biliary PoCUS than staff. However, their confidence in the test was the same as staff. Additionally, they were less willing than staff to operate based on a PoCUS study. We speculate that this may stem from a lack of confidence in their clinical diagnostic skills or from a concern that their staff would require formal imaging before proceeding to operate. However, more research is required to explain these discrepancies.

\section{Conclusion}

Our survey shows an opportunity for improvement in surgical care. Surgeons are missing an opportunity to provide efficient care when biliary disease is identified by emergency physicians. Our study identifies a knowledge translation gap that, if bridged, may lead to more efficient patient care. 
Affiliations: From the Division of Emergency Medicine, Western University, London, Ont. (Thompson, Myslik); the Department of Surgery, Western University, London, Ont. (Koichopolos, Leeper); the Division of Critical Care Medicine, Western University, London, Ont. (Leeper); and the Schulich School of Medicine and Dentistry, Western University, London, Ont. (Vandelinde).

Competing interests: R. Leeper is an associate editor of $C F S$; he was not involved in the review of or decision to accept this paper for publication. No other competing interests were declared.

Contributors: All authors contributed substantially to the conception, writing and revision of this article and approved the final version for publication.

\section{References}

1. Everhart JE, Khare M, Hill $M$ et al. Prevalence and ethnic differences in gallbladder disease in the United States. Gastroenterology. 1999:117:132-9.

2. Urbach, D, Stukel, TA. Rate of elective cholecystectomy and the incidence of severe gallstone disease. CMA7 2005;172:1015-19.

3. Hilsden, R, Leeper, R, Koichopolos, J, et al. Point of care biliary ultrasound in the emergency department (bused): implications for surgical referral and emergency department wait times. Trauma Surg Acute Care Open 2018;3:e00164.

4. Summers SM, Scruggs W, Menchine MD et al. A prospective evaluation of emergency department bedside ultrasonography for the detection of acute cholecystitis. Ann Emerg Med 2010:56:114-22.

5. Murphy PB, Paskar D, Hilsden R et al. Acute care surgery: a means for providing cost-effective, quality care for gallstone pancreatitis. World f Emerg Surg 2017;12:20.

6. Gustafsson C, Lindelius A, Torngren S et al. Surgeon-performed ultrasound in diagnosing acute cholecystitis and appendicitis. World $\mathcal{F}$ Surg 2017;42:3551-9. 